\title{
Research of Dynamic Characteristic of Belt Conveyor
}

\author{
Jun-Xia LI ${ }^{1,2}$, Xiao-Xu PANG ${ }^{3}$, Yu-Jin LI ${ }^{4}$ \\ 1College of Mechanical Engineering, Taiyuan University of Technology, Taiyuan 030024, China; \\ 2Mine Fluid Control Engineering research Center(Laboratory) in Shanxi Province, \\ Taiyuan 030024, China; \\ 3 Henan University of Science and Technology, Luoyang 471023, China; \\ 4Beijing HuaYu Engineering Co.Ltd of Sino-coal International Engineering Group, \\ Peking 100120, China
}

Keywords: Belt conveyor, Dynamic characteristic, Starting time, Natural frequency.

\begin{abstract}
Aiming at the dynamic question of the belt conveyor, based on the Kelvin-Voigt viscoelastic constitutive law the longitudinal dynamic governing equation of the viscoelastic conveyor belt was established. The generalized coordinate method was adopted to solve dynamic displacement and dynamic tension of the weight tension belt conveyor at the end. According to the longitudinal dynamic model the natural frequency and the dynamic tension were investigated by the research of digital simulation. The results show that the natural frequency of the belt conveyor will become small when the throughput increases. Moreover, increasing the value of the starting time will lead to a deceasing in dynamic tension and then reduce the design safety coefficient and the cost of production.
\end{abstract}

\section{Introduction}

Belt conveyor is a viscoelastic body with driving mechanism and bearing mechanism as a whole that the longitudinal length has about ten kilometers. In the design of belt conveyor, we usually ignore vibration and impact in the belt, but to take static design into account. In order to ensure the safety of the operation of the conveyor, we often increase the safety factor of its design, which also increases the production costs of conveyor. To reduce production cost and optimize performance, at home and abroad, many research institutions have carried out the dynamic analysis of large belt conveyor [1-2]. In the early 1960s, the Soviet Union began to study the dynamics of the conveyor. The starting characteristics of the constant acceleration or AC motor series resistance are studied by using the impulse principle [3]. Based on theory of travelling wave, which was found by Hannover in Germany in 1973, and the dynamic characteristics of the conveyor was studied [4-5].The lateral vibration of the belt conveyor was studied in Shandong University of Science and Technology, Xi'an University of Science And Technology and Taiyuan University of Science and Technology. The relationship between the lateral vibration and the speed and tension of the belt conveyor is obtained, which can provide a theoretical basis for the development of belt conveyor [10-11].

The study above is based on the discrete conveyor model. Although its analysis is aimed at viscoelastic model, in the process of analysis, it is simplified to the elastic model, which is not able to carry out more practical overall dynamic research. In this paper, a continuous dynamic equation on tail hammer tensioning of the belt conveyor is established on the basis of Kelvin-Voigt model. The viscoelastic continuity equation is solved by using the generalized coordinate method. Analysis of the natural frequency of the conveyor and the longitudinal dynamic tension of the conveyor, provides a more accurate method for the dynamic characteristic of belt conveyor.

Nomenclature

$\begin{array}{lc}\sigma(t) & \text { stress of conveyor belt } \\ E & \text { elastic modulus of belt } \\ \varepsilon & \text { strain } \\ \mu & \text { viscous hysteresis coefficient } \\ A & \text { cross section area of belt } \\ c & \text { elastic wave propagation velocity }\end{array}$




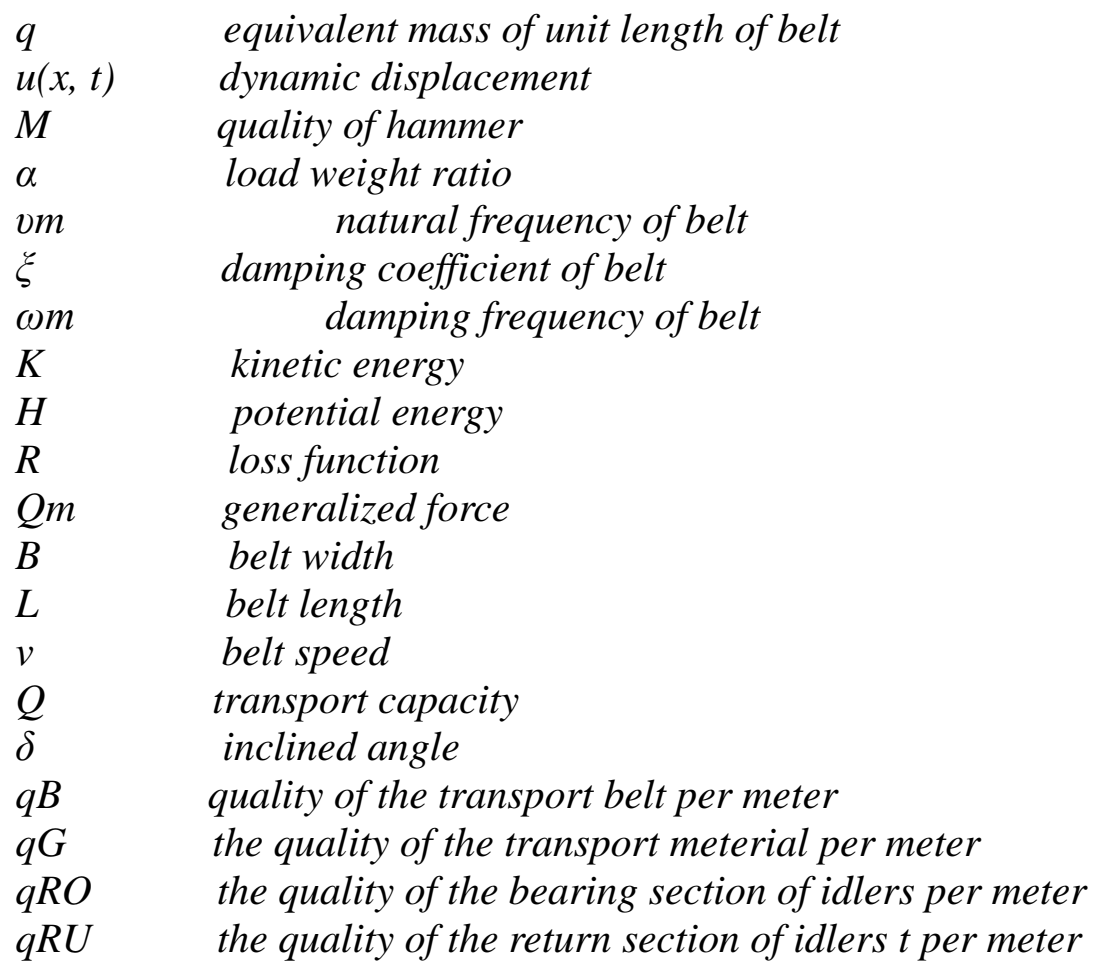

\section{Continuous Dynamic Equation of Belt Conveyor}

In this paper, the inertia, elasticity and damping of the conveyor belt with viscoelastic characteristics are set to be the continuous. The movement of the conveyor system is described by the partial differential equation.Fig. 1 is a diagram of belt conveyor. In order to establish the mathematical model of conveyor, the following hypothesis is made: Neglect transverse vibration impact because it is small by comparing with longitudinal vibration; Neglect shear and bending stress of conveyor; Neglect the change of belt length which is caused by the longitudinal degree of the conveyor belt; Neglect lateral deformation which is caused by longitudinal tension of the belt.

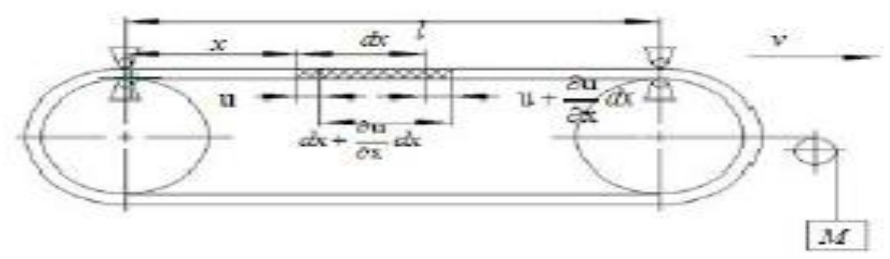

Figure 1.Schematic diagram of the belt conveyor

The conveyor belt is made up of rubber and fabric core, which has both elastic and viscous characteristics. In this paper, Kelvin-Voigt as the dynamic model is used to study and the constitutive law is

$$
\sigma(t)=E\left(1+\mu \frac{\partial}{\partial t}\right) \varepsilon
$$

Where $\sigma(\mathrm{t})$ - Stress of belt;

E- Elastic modulus of belt;

$\varepsilon$ - strain;

$\mu-$ Viscous hysteresis coefficient.

Tension of the belt at $\mathrm{x}$ is

$$
\mathrm{S}(x, t)=\mathrm{EA}\left(1+\mu \frac{\partial}{\partial t}\right) \frac{\partial u}{\partial x}
$$


Where A-Cross section area of belt.

Under unstable conditions, especially the starting and braking process, the belt isn' $t$ only effected by static tension, but also effected by dynamic tension which is caused by speed change of the conveyor. Dynamic displacement $\mathrm{u}=\mathrm{u}(\mathrm{x}, \mathrm{t})$ is due to dynamic tension. In this paper, we mainly study the dynamic characteristics of the conveyor, and ignore the static displacement caused by the static tension.

The longitudinal vibration of the conveyor can be neglected, and the dynamic equation of the viscoelastic longitudinal vibration of the conveyor is

$$
\frac{\partial^{2} u}{\partial t^{2}}=c^{2}\left(1+\mu \frac{\partial}{\partial t}\right) \frac{\partial^{2} u}{\partial x^{2}}-a(t)
$$

Where c-Elastic wave propagation velocity, $c=\sqrt{\frac{E A}{q}}$

$\mathrm{q}$-Equivalent mass of unit length of belt.

The boundary and initial conditions of tail hammer tension is following.

A boundary condition is

$$
\mathrm{u}(0, \mathrm{t})=0 \frac{M}{2} \frac{\partial^{2} u(l, t)}{\partial t^{2}}=-E A\left(1+\mu \frac{\partial}{\partial t}\right) \frac{\partial u(l, t)}{\partial x}
$$

Where M-The quality of the hammer.

$\mathrm{B}$ initial condition is

$$
u(x, 0)=0 \quad \frac{\partial u(x, 0)}{\partial t}=0
$$

When the conveyor starts up, displacement and speed of the conveyor are zero in the whole belt.

\section{Viscoelastic Longitudinal Vibration Analysis}

\section{Homogeneous Equation}

Eq.(3) is a non homogeneous viscoelastic vibration equation. In order to get its analytical solution, and it needs to be homogeneous to obtain the mode function. $\mathrm{u}(\mathrm{x}, \mathrm{t})$ can be solved by the homogeneous and separation of variables.

set up

$$
u(x, t)=X(x) T(t)
$$

Where $X(x)$ is a function of $x$ coordinates, $T(t)$ is a function of $t$ coordinates. Set Eq. (6) into Eq. (3) which can be obtained by the homogeneous equation

$$
\frac{\ddot{T}}{c^{2}(T+\mu \dot{T})}=\frac{X^{\prime \prime}}{X}=-\lambda^{2}
$$

By the boundary condition of the longitudinal vibration and the normalized condition, the vibration mode function is

$$
\mathrm{X}_{\mathrm{m}}=b \sin \lambda_{m} x=b \sin \frac{\eta_{m} x}{l}
$$

Where a-The equivalent mass of belt and half of hammer mass ratio (load weight ratio)

$$
\alpha=\frac{2 q l}{M}=\eta \tan \eta
$$

$\mathrm{b}$ - Constant, determined by the initial condition.

Where $\alpha=\eta \tan \eta$ is transcendental equation, which has an infinite number of solutions, Table 1 shows value of $\eta$ corresponding to different $\alpha$. 
Table 1. Value of $\eta$ corresponding to different $a$

\begin{tabular}{|c|c|c|}
\hline$\alpha$ & $\eta_{1}$ & $\eta_{2}$ \\
\hline 0.01 & 0.10 & 3.15 \\
\hline 0.4 & 0.60 & 3.26 \\
\hline 0.8 & 0.79 & 3.37 \\
\hline 1.0 & 0.86 & 3.43 \\
\hline 2.0 & 1.08 & 3.64 \\
\hline 4.0 & 1.27 & 3.94 \\
\hline 10 & 1,43 & 4.31 \\
\hline 100 & 1.56 & 4.67 \\
\hline 1000 & 1.57 & 4.71 \\
\hline
\end{tabular}

By Eq. (7), we can conclude

$$
\mathrm{T}=e^{-\xi \omega_{\mathrm{m}} t}\left(\mathrm{D}_{1} \cos \omega_{m} t+\mathrm{D}_{2} \sin \omega_{m} t\right)
$$

Where vm-Natural frequency of belt ; $v_{m}=\frac{c \eta_{m}}{l}$

$\xi$-Damping coefficient of belt, $\xi=\frac{\mu v_{m}}{2}$;

$\omega \mathrm{m}$-Damping vibration frequency of belt, $\omega_{m}=v_{m} \sqrt{1-\xi^{2}}$

From vm and table 1, it shows that the natural frequency of the conveyor increases with $\alpha$ value, but the damping frequency and $\alpha$ do not have the linear relationship.

The subscript $\mathrm{m}$ of $\mathrm{vm}$ means that for an arbitrary natural number, a corresponding natural frequency is obtained, and it is the natural frequency. It can be seen from $v_{\mathrm{m}}$ that the natural frequency of conveyor is not unique, but an infinite number of discrete values. The natural frequency does not exist between two arbitrary discrete values. While $m=1$, natural frequency is the fundamental frequency. The fundamental frequency vibration is the dominant position in the system of free vibration and forced vibration. When a device starts up, the fundamental frequency is the first frequency of resonance. Fundamental frequency is the primary task on the vibration of belt conveyor.

\section{Non Homogeneous Equation}

The vibration of belt is caused by the unstable condition. In this paper, the generalized coordinate method is used to solve the Eq.(3). The displacement at any cross section of belt under unstable condition is

$$
u(x, t)=\sum_{m=1}^{\infty} X_{m}(x) q_{m}(t)
$$

From the above, $\mathrm{X}(\mathrm{x})$ is mode of vibration which is solved by homogeneous equation, that is $X_{m}(x)=\sin \frac{\eta_{m} x}{l}$. equation must be used.

The equation is

$$
\frac{d}{d t} \frac{\partial K}{\partial \dot{q_{m}}}-\frac{\partial K}{\partial q_{m}}+\frac{\partial R}{\partial \dot{q_{m}}}+\frac{\partial H}{\partial \boldsymbol{q}}=Q_{m}
$$

Where $\mathrm{K}$ - kinetic energy;

$\mathrm{H}$ - potential energy;

$\mathrm{R}$-Loss function (to determine mechanical energy reduction), $\mathrm{R}=-\frac{1}{2} \frac{d}{d t}(K+H)$;

Qm-Generalized force corresponding to generalized coordinates. 
Dot from qm denotes differentiation of the generalized coordinates with respect to time. Meanwhile, generalized velocity is the time derivative of generalized coordinates.

The kinetic energy of the conveyor system is equal to kinetic energy of the conveyor belt's equivalent mass plus kinetic energy of load. Kinetic energy of conveyor system is

$$
\mathrm{K}=\frac{q}{2} \int_{0}^{l} X^{2} \sum_{n=1}^{\infty} q_{m} d x+\frac{M}{2}\left[\sum_{n=1}^{\infty} X^{2} \dot{q}_{m}^{2}\right]
$$

Potential energy of conveyor system is

$$
\mathrm{H}=\frac{E \mathrm{~A}}{2} \int_{0}^{l}\left[\sum_{n=1}^{\infty} X^{\prime}(x) q_{m}(t)\right]^{2} d x
$$

The loss function depends on the internal resistance of conveyor belt. Because the belt is viscoelastic, it can be gotten by the orthogonality.

$$
R=\frac{\mu E \mathrm{~A}}{2} \sum_{n=1}^{\infty} \dot{q}_{m}^{2} \int_{0}^{l} X^{\prime} d x
$$

Set Eq. (11), Eq.(12) and Eq.(13) into Eq. (10), and rearrange as

$$
\begin{aligned}
& \ddot{q}_{m}+\frac{\mu c^{2} \int_{0}^{l} X^{\prime 2} d x}{\int_{0}^{l} X^{2} d x+\frac{M}{q} X^{2}(l)} \dot{q}_{m}+\frac{c^{2} \int_{0}^{l} X^{\prime 2} d x}{\int_{0}^{l} X^{2} d x+\frac{M}{q} X^{2}(l)} q_{m} \\
& =\frac{Q_{m}}{q \int_{0}^{l} X^{2} d x+M X^{2}(l)}
\end{aligned}
$$

Simplifying Eq. (7) and the boundary condition, we have

$$
\ddot{\mathrm{q}}_{m}+\mu c^{2} \lambda^{2} \dot{q}+c^{2} \lambda^{2} q_{m}=-\frac{\lambda^{2} Q_{m}}{q \int_{0}^{l} X^{\prime 2} d x}
$$

Eq.(15) is suitable for various boundary conditions (such as fixed winch, constant tension). But under different boundary conditions, $\mathrm{X}(\mathrm{x})$ has different modes.

Deadweight and Hammer force act on a micro unit dx. We can conclude from principle of virtual work

$$
\mathrm{Q}_{m}=\mathrm{M} a(t) X_{m}(l)+\int_{0}^{l} q a(t) X_{m} d x=\frac{\alpha M}{\eta_{m}} a(t)
$$

Set Eq.(16) into Eq. (15), to give

$$
u(x, t)=\frac{q l^{2}}{E \mathrm{~A}} H_{m} \sum_{m=1}^{\infty} \frac{\sin \frac{\eta_{m} x}{l}}{\eta_{m}\left(1-\xi^{2}\right)} A(t)
$$

Where $H_{m}=\frac{4}{\eta_{m}\left(2 \eta_{m}+\sin 2 \eta_{m}\right)}$

Setting (17) into the Eq. (2), then dynamic tension of belt is

$$
\begin{gathered}
\mathrm{A}(t)=\omega_{m} \int_{0}^{t} e^{-\xi v_{m}(t-\tau)} a(\tau) \sin \omega_{m}(t-\tau) d \tau \\
s(x, t)=q l \sum_{m=1}^{\infty} \frac{H_{m}}{1-\xi^{2}} \cos _{l} \eta_{m} x[A(t)+\mu \dot{A(t)}]
\end{gathered}
$$

From Eq. (17) and Eq. (18), we can conclude that the maximum displacement of belt takes place on the tension roller, and the maximum dynamic tension occurs at the input point of driving roller.

\section{Effect of Load Ratio on Natural Frequency}

From Eq.(8) and Table 1, we can conclude that, with the increase of load ratio, the natural 
frequency of the conveyor is increasing but there is no linear relationship. There are two kinds of limit conditions for the natural frequency of the conveyor.

(1)When the hammer quality is smaller than the equivalent mass of conveyor belt, it means as $\alpha=\infty$, then $\eta=\frac{2 n-1}{2} \pi(n=1,2 \cdots)$. Based on this, we can conclude $v_{m}=\frac{c}{l} \frac{2 n-1}{2} \pi$. At this time, the vibration frequency of the system is the largest.

(2)When the hammer quality is larger than the equivalent mass of conveyor belt, it means

as $\alpha=0$, then we can conclude $v_{m}=\frac{c}{l} \sqrt{\alpha}$. At this time, the free vibration frequency of the conveyor is almost zero. This situation almost does not exist, so that the vibration of the conveyor will always exist.

\section{Case Analysis}

Main parameters of belt conveyor are shown in Table. 2.

Table 2. Main parameters of belt conveyor

\begin{tabular}{|c|c|}
\hline Nomenclature & parameter \\
\hline Belt length $L / \mathrm{m}$ & 1504 \\
\hline Belt width $B / \mathrm{m}$ & 0.8 \\
\hline Belt speed $\nu /(\mathrm{m} / \mathrm{s})$ & 3.15 \\
\hline Transport capacity $Q /(\mathrm{t} / \mathrm{h})$ & 257 \\
\hline Inclined angle $\delta /\left(^{\circ}\right)$ & 0 \\
\hline Type & ST1250 \\
\hline Belt thickness $/ \mathrm{m}$ & 0.017 \\
\hline Quality of the transport belt per meter $q_{B} /(\mathrm{kg} / \mathrm{m})$ & 24.7 \\
\hline Quality of the transport material per meter $q_{G} /(\mathrm{kg} / \mathrm{m})$ & 22.68 \\
\hline Quality of the bearing section of idlers per meter $q_{R o} /(\mathrm{kg} / \mathrm{m})$ & 8.8 \\
\hline Quality of the return section of idlers t per meter $/(\mathrm{kg} / \mathrm{m})$ & 2.93 \\
\hline Hammer quality $(\mathrm{kg})$ & 1750 \\
\hline
\end{tabular}

\section{Natural Frequency of the System}

Eq.(8) shows that there is a close relationship between the natural frequency and hammer quality. By the above parameters and Eq.(8), full load of bearing strand, empty load of bearing strand and the first three order natural frequency of return strand are shown in Tab. 3. It is known that the natural frequency of full load is the smallest, so the natural frequency of the bearing load strand should be avoided in resonance analysis.

Table.3 First three order natural frequency of the carrying strand and return strand

\begin{tabular}{|c|c|c|c|}
\hline Nomenclature & $v_{1}$ & $v_{2}$ & $v_{3}$ \\
\hline Full load of bearing strand & 1.48 & 4.45 & 7.45 \\
\hline Empty load of bearing strand & 1.82 & 5.51 & 9.32 \\
\hline Empty load of return strand & 1.97 & 6.0 & 10.21 \\
\hline
\end{tabular}

\section{Tension Response Characteristics of Different Starting Time}

Eq.(18) shows that at input point $(\mathrm{x}=0)$ of driving roller, dynamic tension has the maximum value. In the case of the above parameters, this paper analyzes the dynamic tension input point $(x=0)$ of driving roller, dynamic load coefficient and strain curve respectively corresponding to starting time of 60s, 120s and 180s. Dynamic load coefficient defines as dynamic tension plus static tension, and then divided by static tension.

As can be seen from Fig.2, when the starting time is 60 s, 120s, and 180 s, tension peak at input 
point of driving roller respective is $6.4 \times 104 \mathrm{~N}, 6.0 \times 104 \mathrm{~N}$ and $5.8 \times 104 \mathrm{~N}$. Stable value is about $5.5 \times 104 \mathrm{~N}$. Moreover, dynamic tension respectively reduces $16.4 \%, 9.1 \%$ and $5.5 \%$. It can be known that increasing the starting time that is to reduce the starting acceleration can effectively reduce dynamic tension. It provides a theoretical basis for reducing design safety factor. As shown in Fig. 2, the tension respectively reaches the first peak at $19 \mathrm{~s}, 60 \mathrm{~s}$ and $30 \mathrm{~s}$. It is because the start time is shorter, the input speed is the greater. The large speed wave can cause that the influence of running resistance on speed wave is smaller. Therefore, when the starting time is $60 \mathrm{~s}$, the time of the first peak is shortest.

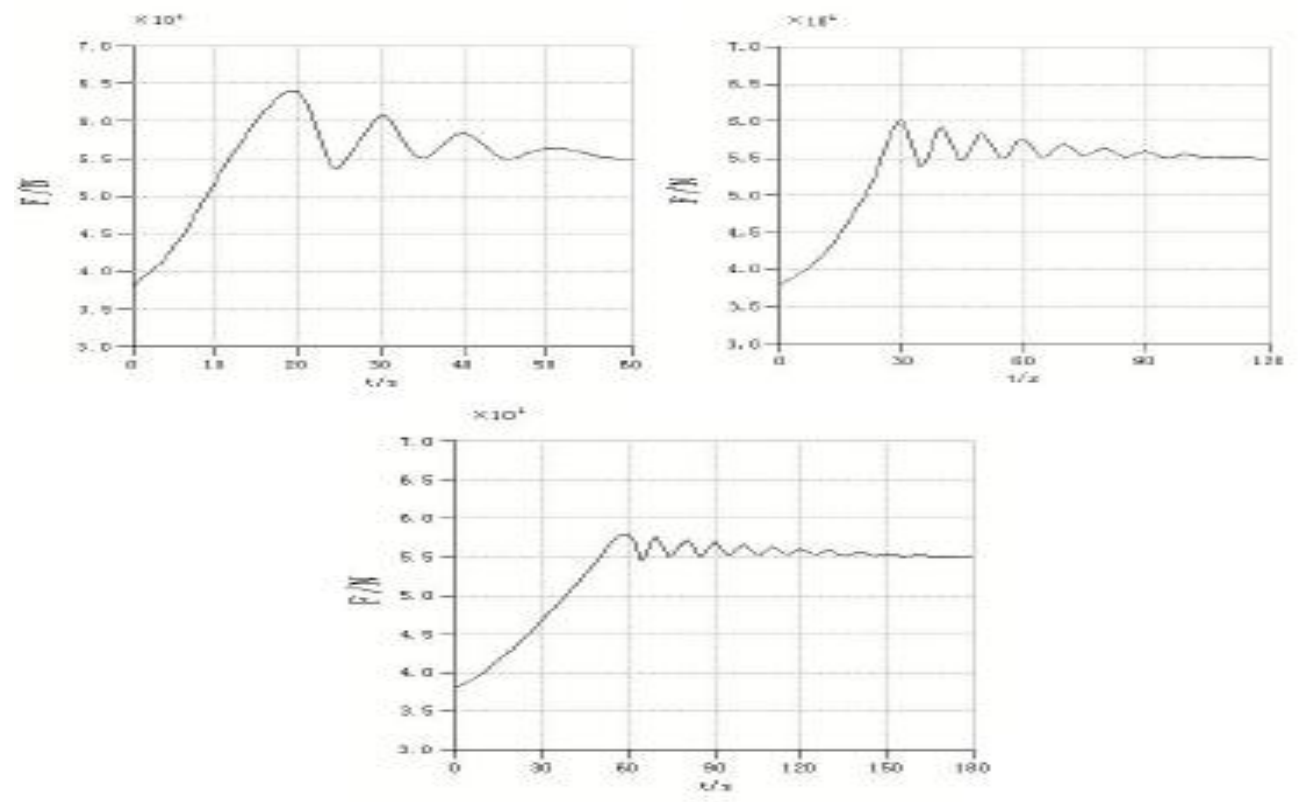

Figure 2. Tension at the meeting point of drive drum at different starting time

Fig. 3 and Fig. 4 respectively correspond to dynamic loading coefficient curve $\mathrm{K}$ and strain curve at different starting time. When the dynamic load coefficient is 1.16, 1.05 and 1.09 respectively, and the maximum strain is respective $0.0032,0.00289$ and 0.0030 . The attenuation trend of the curve and the attenuation trend of the tension are basically consistent, and the peak time is also basically consistent. It further confirms that increasing start time can effectively reduce starting dynamic tension.
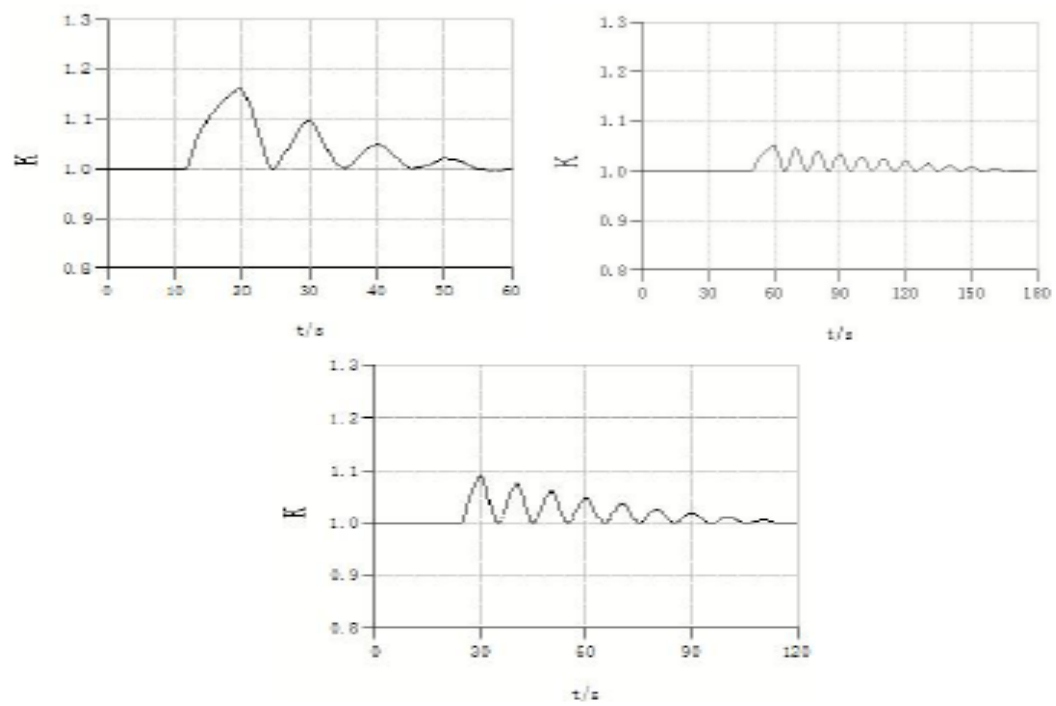

Fig.3 Dynamic loading coefficient $\mathrm{K}$ at the meeting point of drive drum at different starting time 

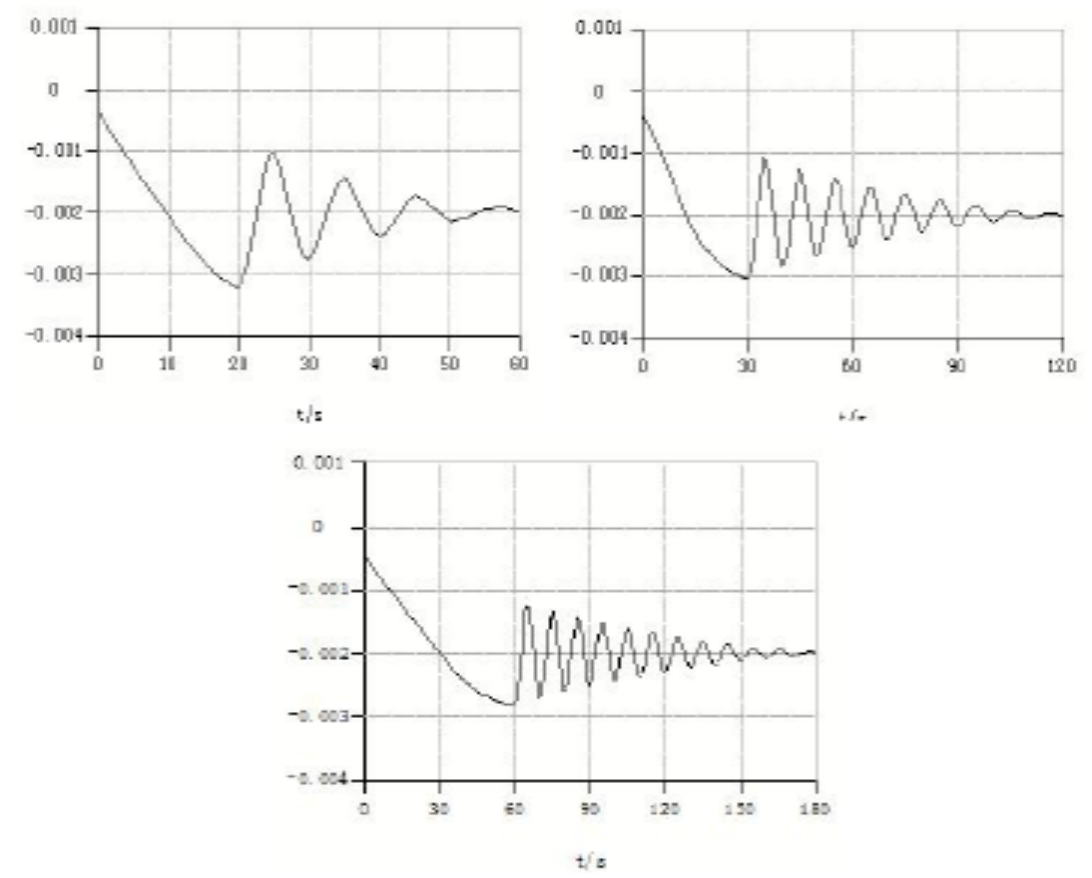

Fig.4 Strain at the meeting point of drive drum at different starting time

From the above analysis, it is known that with starting time extending, the dynamic tension of belt is reduced by $9.375 \%$ which is equal to the reduction of the dynamic tension.

\section{Conclusion}

(1) Based on Kelvin-Vogit model, the partial differential equation of the belt conveyor is established. Moreover, dynamic equation of the longitudinal vibration of the conveyor is obtained accurately.

(2) The analytical solution of dynamic displacement and dynamic tension of belt is derived by using the generalized coordinate method. The relationship between the natural frequency of conveyor and the load ratio is established. With the load ratio increasing that is constantly increasing transport capacity, the natural frequency of conveyor is gradually reduced.

(3) Simulation shows that increasing the starting time, that is to reduce the starting acceleration, can effectively reduce the starting dynamic tension and dynamic load coefficient, so as to provide a theoretical basis for the dynamic design of the conveyor.

\section{Acknowledgment}

The research are supported by the NSFC- Shanxi coal based low carbon fund (Grant No.U1510205) and the scientific and technological project in Shanxi Province (Grant No. 2015031006-2) .The first author gratefully acknowledged the helpful discussions with the research group and colleagues of Taiyuan University of technology.

\section{Reference}

1. Wang Baohua, Liu Jingping, Lu Shan. Analysis on Development of Large-power and High-speed Belt Conveyors [J].Mining \& Processing Equip-ment. 42(1), 4 -7 (2014)

2. Xi Pingyuan, Zhu Jiang, Shentu Liufang. Analysis and PID Control on Dynamical Performance of Conveyor Possessing Closed-loop [J]. Transactions of the Chinese Society for Agricultural Machinery. 41(12), 211-216 (2010)

3. He Tiancai. The dynamic Analysis and the study of Optimized Control on the Belt Conveyor 
in Sihe Mine [D]. China University of Mining \& Technology, Beijing (2009)

4. Ghayesh M.H, Khadem S.E. Non-linear vibration and stability analysis of a partily supposed conveyor belt by a distributed viscoelastic foundation [J]. Structural Engineering and Mechani. 27(1), 17-32 (2007)

5. H. Funke, K. Hanrtmann, H. Lauhoff. Designed Operating Performance of a Long-Distance Belt Conveyor System with Horizontal Curves and simultaneous Material Transport in the Upper and Lower Strands [J]. Bulk Solids Handling. 20(1), 45-55 (2000)

6. Hu Ding, Li-Qun Chen. Nonlinear models for transverse forced vibration of axially moving viscoelastic beams [J]. Shock and Vibration. 18(1-2), 281-287 (2011)

7. Guo Sheng, Liu Jian, Li Zhiren, DongLi, HUANG Wei. Experimental research on air film formation behavior of air cushion belt conveyor with stable load [J]. Science China (Technological Sciences), 06, 1424-1434 (2013)

8. Nordell L.K.Overland Conveyors Designed for Efficient Cost and Performance [J]. Bulk Solids Handling. 26(1), 44-53 (2006)

9. Li Xin. The Dynamic Simulation and software development of belt conveyor system [D]. Taiyuan University of Science \& Technology (2014)

10. Su Peng. Research on the Starting and Barking Integration Automatic Control System of Belt Conveyor [D]. Taiyuan University of Science and Technology (2010)

11. Yin Lianmin. The Research on Control System of the VFD Conveyor Concerning the Automatic Energy-saving Operation [D]. Xi'an University of Science and Technology (2011)

12. Chen Qiuhong. Large Belt Conveyor Dynamics Modeling and Software Development [D]. LiaoNing Technical University (2011)

13. Pang Xiaoxu, Li Yujin, Li Junxia, et al. Analysis on dynamic tension and non-slipping of belt conveyor based on the wave theory [J]. Coal Science and Technology. 04, 92-95 (2015)

14. Zuo Bo, Song Weigang, Wan Liangliang. Comparative analysis on start-up "S-shaped curve" of belt conveyors based on traveling wave theory [J]. Mining \& Processing Equipment. 39(10), 46-52 (2011)

15. Song Weigang, Wang Dan, Zuo Bo. Semi-analytical solution to dynamic continuous model of belt conveyor with single drive [J]. Journal of China Coal Society. 3(6), 217-223 (2012)

16. Mao Jun, Li Xiaojing, Chen Hongyue,et al. Research of the Rigid-flexible Coupling Dynamic Characteristic of the Conveyoring Belt of Belt Conveyor[J]. Journal of Mechanical Transmission. 04, 19-23 (2015)

17. Yuan Hongbing, Kang Shilei. Simulation Method of Belt Conveyor Start-up Phase Based on RecurDyn [J]. Coal Mine Machinery. 01, 107-109 (2015)

18. Meng Jie. The Research on Comprehensive experimental system [D]. Taiyuan University of Science and Technology (2014) 